
allemande

46-1 | 2014

Les fondements normatifs de l'État constitutionnel moderne en Allemagne. Une approche pluridisciplinaire

\title{
Les élections législatives allemandes vues du Parlement européen
}

Foussenatou Ouro

\section{OpenEdition \\ Journals}

Édition électronique

URL : https://journals.openedition.org/allemagne/1450

DOI : $10.4000 /$ allemagne. 1450

ISSN : 2605-7913

Éditeur

Société d'études allemandes

Édition imprimée

Date de publication : 30 juin 2014

Pagination : 243-256

ISSN : 0035-0974

Référence électronique

Foussenatou Ouro, "Les élections législatives allemandes vues du Parlement européen », Revue d'Allemagne et des pays de langue allemande [En ligne], 46-1 | 2014, mis en ligne le 29 juillet 2019, consulté le 22 mai 2021. URL : http://journals.openedition.org/allemagne/1450 ; DOI : https://doi.org/ 10.4000/allemagne. 1450 


\section{Les élections législatives allemandes vues du Parlement européen ${ }^{(1)}$}

\section{- Foussenatou Ouro*}

\section{« L'européanité » de l'Allemagne commentée}

Les spécialistes ont pu dire autrefois de l'Allemagne qu'elle était un géant économique mais un nain politique. Vingt ans après la chute du Mur, la situation a bien changé. L'Allemagne a su se positionner et se réinventer dans une Union qui s'élargit sans cesse $^{(2)}$. Au sein de l'Union, elle est le pays le plus peuplé avec 82 millions d'habitants. Elle dispose de 99 sièges au Parlement européen, de 29 voix au Conseil européen et est le premier pourvoyeur du budget de l'ensemble de l'Union européenne.

Cependant, elle a semble-t-il abandonné cette retenue qui caractérisait jusqu’alors sa politique extérieure, pour exercer un leadership politique de fait, dans une Europe qui se cherche encore. En tant que grande puissance en Europe, l'Allemagne sert également de pont non seulement entre la partie occidentale et orientale du continent européen mais

Doctorante à l'Université de Strasbourg.

1 Eurodéputés ayant apporté leurs témoignages pour cette enquête: Évelyne Gebhardt, groupe de I'Alliance Progressiste des Socialistes et Démocrates au Parlement européen, Allemande, membre du SPD, née le 19 janvier 1954 à Montreuil-sous-Bois (France), rencontrée le 11.09.2013; Alain Lamassoure, groupe du Parti Populaire Européen (démocrates-chrétiens), Français, membre de I'Union pour un Mouvement populaire, né le 10 février 1944 à Pau, rencontré le 11.09.2013; Jean-Paul Gauzes, groupe du Parti Populaire Européen (démocrates-chrétiens), Français, membre de l'Union pour un Mouvement populaire, né le $1^{\text {er }}$ octobre 1947 à Toulouse, rencontré le 11.09.2013 ; Jean-Louis Cottigny, groupe de l'Alliance Progressiste des Socialistes et Démocrates au Parlement européen, Français, membre du Parti socialiste, né le 12 septembre 1950 à Hesdin, rencontré le 12.09.2013; Marc Tarabella, groupe de l'Alliance Progressiste des Socialistes et Démocrates au Parlement européen, Belge, membre du Parti socialiste, né le 11 mars 1963 à Ougrée, rencontré le 08.10.2013; Daniel Cohn-Bendit, groupe des Verts/Alliance libre européenne, coprésident de France Europe Écologie, né le 4 avril 1945 à Montauban, rencontré le 08.10.2013.

2 Olivier Breton et Pierre JAnin, Quand l'Allemagne s'est réinventée. Le choc de la réunification, Paris, Éditions Autrement, 2010, p. 7-9. 
aussi entre l'Europe et les autres puissances de la planète ${ }^{(3)}$. Elle est devenue un modèle pour les pays du Sud qui n'ont pas véritablement pris en compte l'évolution du monde pour bâtir une économie solide. De ce fait, elle est perçue comme un État providentiel et se pose d'emblée comme le principal allié dans le processus de construction de l'Union. Une fierté nationale et internationale est ainsi apparue mais dans un contexte nouveau. Et la vocation internationale du pays s'affirme de plus en plus notamment à travers la construction européenne.

Toutefois, cette évolution ne traduit pas tant la volonté pour l'Allemagne d'affirmer une quelconque prééminence politique au sein de l'Union, puisqu'elle n'a conquis sa pleine souveraineté qu'en $1990^{(4)}$. Le fait qu'elle tienne désormais à jouer pleinement son rôle dans le gouvernement partagé de l'Union, découle de l'émergence ${ }^{(5)}$ d'une nouvelle identité qui se nourrit de l'expérience des épreuves surmontées mais surtout dans la confiance acquise dans la capacité de ses dirigeants à ériger un système économique, social, et démocratique solide et stable qui résiste au choc d'un monde de plus en plus incertain.

Ainsi, s'affirme-t-elle de nos jours entièrement souveraine car consciente de son acquis, de son expérience mais aussi fière de ses principes, de ses valeurs et confiante dans sa capacité à proposer des choix aux Européens et à en assumer la responsabilité. Cette nouvelle attitude de l'Allemagne interpelle et suscite des questionnements puisqu'en même temps, l'identité allemande prend une allure austère et déconcertante. Les différentes crises auxquelles elle est confrontée, ses positions géopolitiques et les défaillances européennes semblent la perturber. Bien qu'elle soit la quatrième puissance mondiale, le pays vit parfois mal les échecs de sa cohésion sociale, les difficultés à intégrer de nouveaux flux migratoires et son rôle de géant européen circonspect ${ }^{(6)}$.

Quel est donc en effet le jugement à porter sur la position de l'Allemagne face à l'Union européenne et quel espoir d'avenir pourrait-on formuler vis-à-vis d'elle au vu de sa réussite économique dans l'actuel régime, alors que certains citoyens allemands appellent à la retenue de la politique d'intégration européenne? L'élection législative d'automne dernier a été ainsi très importante non seulement pour l'Allemagne mais aussi pour la France et pour l'Europe.

3 Jacques-Pierre Gougeon, L'Allemagne du xxI siècle, une nouvelle nation? Éléments de réponse, Paris, Armand Colin, 2009, p. 8-22: "Tous ces attributs font de l'Allemagne une puissance difficilement contestable. Troisième puissance économique du monde, talonnée par la Chine, avec un PIB supérieur de $35 \%$ à celui de la France et qui représente $6 \%$ du PIB mondial. Première puissance commerciale de la planète avec 9,2\% des exportations mondiales en marchandises et services (soit $45 \%$ de son PIB). Première puissance économique de l'Union européenne dont elle représente $27 \%$ de la richesse de la zone euro. Premier contributeur au budget de l'Union européenne à hauteur de $20 \%$, deuxième contributeur au budget de l'OTAN et troisième à celui des Nations unies. En outre, l'Allemagne est en chiffres absolus, le deuxième donateur d'aide au développement derrière les États-Unis. »

4 J.-P. Gougeon, L'Allemagne du XXI siècle (note 3), p. 15: «...Angela Merkel s'appuyant sur le travail effectué par son prédécesseur fait observer que l'Allemagne plus grand pays d'Europe doit s'affirmer et s'imposer dans de nombreux domaines. »

5 Isabelle Bourgeois (dir.), Allemagne, Les chemins de l'unité. Reconstruction d'une identité en douze tableaux, Cergy-Pontoise, Cirac, 2011, p. 7-11.

6 Jean-Louis de la VAissière, Qui sont les Allemands?, Paris, Max Milo Éditions, 2011, p. 209. 
Puissance incontestable, jouant un rôle moteur dans l'Union européenne, l'Allemagne a compris qu'elle devait respecter un certain nombre de critères économiques et budgétaires fondamentaux et résiste très bien à la crise. Cependant, si on peut parler de la réussite économique allemande, compte tenu des résultats obtenus en matière de croissance, de compétitivité et de lutte contre le chômage, il n'en reste pas moins que bien d'autres paramètres ne sont pas encore stabilisés. L'Allemagne reste par exemple confrontée au problème du vieillissement de sa population, lequel requiert de l'Allemagne une prompte réaction pour y remédier.

Dans le présent article, il nous a donc semblé intéressant de comparer les différentes réactions qui se sont manifestées à l'occasion des élections législatives allemandes du 22 septembre 2013, afin de mieux comprendre la perception que les acteurs de la politique européenne ont de la place qu'occupe l'Allemagne dans le processus de construction de l'Union. L'objectif assigné étant d'aider, sans parti pris, à en saisir les enjeux, à surmonter les préjugés et à alimenter un regard nouveau sur l'Allemagne afin de saisir comment elle a su élaborer sa nouvelle identité et les valeurs qui font sa force aujourd'hui.

\section{Le regard des eurodéputés sur la place de l'Allemagne au sein de l'Union européenne}

À partir de l'approche mise en exergue, les formes narratives, les registres dans lesquels s'inscrivent les propos exprimés par les eurodéputés et la représentation qu'ils se font d'eux-mêmes, sont inhérents à leur positionnement politique. En effet, ce n'est pas que l'Allemagne se perçoive nécessairement comme étant la première puissance européenne. Pour Alain Lamassoure, eurodéputé du PPE, la position de l'Allemagne tend actuellement plutôt vers un affaiblissement économique, social et politique ${ }^{(7)}$. D’une part, la situation intérieure du pays, par référence aux analyses de Matthias Matussek ${ }^{(8)}$, est affectée par le vieillissement de sa population et les difficultés engendrées par la réforme du système de la protection sociale, les restrictions budgétaires et le cours trop élevé de l'euro. Mais d'autre part, l'Allemagne est également confrontée sur la scène internationale au Royaume-Uni qui ne s'investit que très partiellement dans le processus de construction européenne, refusant d'exercer au sein de l'Union ses responsabilités de grande puissance. L'Allemagne peut être désormais tentée de l'imiter.

L'Italie est également affaiblie, selon Alain Lamassoure, par la médiocrité de son système politique et économique, si bien qu'elle n'a jamais joué un rôle de premier plan au sein de l'Union, bien que s'affichant entièrement pro-européenne. À cela s'ajoute l'affaiblissement économique et politique de la France qui, devenue très compétitive,

7 Les analyses d'Alain Lamassoure rejoignent celles d'Édouard Husson, Une autre Allemagne, Paris, Gallimard, 2005, p. 390, lorsque ce dernier écrit que l'Allemagne est « un pays dont on doit moins redouter la puissance que l'affaiblissement, une nation menacée par son déclin démographique, le cœur industriel fragile d'un continent européen qui risque d'être un grand perdant de la mondialisation ». Voir également Jochen Кивоsсн, "La place de l’Allemagne dans l'Union européenne », in: L'Allemagne Actes 2003, Bordeaux, CRDP d'Aquitaine, 2004, p. 30; J.-P. Gougeon, L'Allemagne du XXI siècle (note 3), p. 33-34.

8 Matthias Matussek, Nous les Allemands, Paris, Éditions Saint Simon, 2007, p. 77-81. 
n’a cependant pas su faire depuis quelques années les réformes nécessaires pour rester au même niveau que l'Allemagne. Que celles-ci concernent les secteurs politico-administratifs, économiques, son système de sécurité sociale ou sa politique en matière de retraite. L'affaiblissement de la France s'est aussi avéré depuis le début du quinquennat de François Hollande, qui feint d'oublier que L'Union européenne est un système d'échange dans lequel on avance avec le modèle franco-allemand. Issus de précieuses proximités acquises de haute lutte, ces deux pays sont parvenus à rassembler tous les autres États européens pour poser les jalons de l'Europe d'aujourd'hui ${ }^{(9)}$.

Mais alors que les autres puissances n'arrivaient pas à moderniser leur système politique, les Allemands ont réussi la réunification et la remise à niveau des nouveaux Bundesländer. Ils ont surmonté les problèmes liés à la réunification avec beaucoup d'abnégation. Ils ont su opérer les réformes qui ont redonné à l'Allemagne la compétitivité qu'elle avait perdue depuis dix ans. Depuis deux ans, l'excédent commercial allemand a dépassé celui de la Chine ${ }^{(10)}$. L’Allemagne est ainsi redevenue plus forte grâce à son nouveau modèle économique et démographiquement plus nombreuse par comparaison aux autres États ${ }^{(11)}$. Peut-on pour autant affirmer que l'Allemagne a déjà su trouver sa propre identité?

Évelyne Gebhardt, eurodéputée allemande du PSE, hésite pour sa part à répondre à cette question. Elle affirme que des efforts ont été faits, même s'ils donnent l'impression que l'Allemagne est toujours à la recherche de sa véritable identité, non seulement en elle-même mais aussi au sein de l'Union européenne. Les mutations connues depuis les années 1990 et le choc des cultures à l'intérieur de l'Allemagne ont été aussi considérables. Il serait donc trop tôt pour conclure que l'Allemagne a trouvé cette identité. Certes, l'Allemagne est un grand pays mais en même temps, celui-ci reste confronté à d'innombrables problèmes sociaux qui ne peuvent laisser indifférents.

Avant 1945, elle a voulu être hégémonique car elle se considérait comme bien supérieure aux autres États. C'est ainsi qu'elle s'est retrouvée dans une folie meurtrière dont on connaît le dénouement ${ }^{(12)}$. Les Allemands ont perdu la guerre mais aujourd'hui, ils gagnent celle de l'économie. L’Allemagne est également le pays qui, dans la situation de crise actuelle, a été la plus sollicitée pour apporter son concours financier à la Grèce. Née de la guerre, elle milite aujourd'hui pour la paix car consciente qu'il n'y a ni race supérieure, ni culture supérieure mais seulement des êtres humain libres et égaux. La question qui reste posée est de connaître le rôle qui lui incombe après la phase de rédemption et de réhabilitation.

Toutefois, sans trop vouloir embellir l'image de son rôle européen, l’Allemagne hésite en effet à assumer un leadership car elle reste consciente des retombées négatives que celui-ci pourrait générer sur son économie. Pendant longtemps, elle a fonctionné sans prendre de responsabilités politiques majeures. À titre d'exemple, elle n’a pas participé

9 Béatrice Angrand, Aurélie Marx, L’Allemagne, Paris, Éditions du Cavalier Bleu (coll. Idées Reçues), 2006, p. 33-45.

10 Propos recueillis de l'eurodéputé Alain Lamassoure.

11 B. Angrand, A. Marx, L'Allemagne (note 9), p. 33-45: « ... c'est le modèle économique (l'économie sociale de marché se reposant sur l'organisation industrielle) mis en place après la deuxième guerre mondiale qui a permis d'avoir ce succès... ». 
à la première guerre du Golf qu'elle pensait pourtant justifiée faute d'une politique étrangère réellement définie ${ }^{(13)}$.

D'ailleurs, on a pu observer pendant toute la crise grecque la prudence d'Angela Merkel, son hésitation à apporter une solution adéquate à cette crise. Une attitude qui se justifiait en partie par la dégradation de la politique intérieure de son pays qu'elle ne souhaitait pas aggraver, bien qu'ayant une solution en mains. La Chancelière était également consciente qu'elle ne pouvait agir en marge du rapport consensuel avec ses autres partenaires de l'Union européenne. Disposant de plusieurs facteurs d'influence qui la rendent solidaire de l'Europe, l'Allemagne finance toute une série de politiques par les fonds de cohésion, lesquels permettent à d'autres États membres d'entreprendre des investissements. Néanmoins, on peut à certains égards lui reprocher son inertie face aux crises portugaise, irlandaise et italienne. Certains ne comprenant pas l'attitude plus favorable de l'Allemagne vis-à-vis de la Grèce et moins vers les autres.

Peut-on pour autant réaffirmer que la réussite de l'Allemagne favorise le succès collectif de l'ensemble des États membres? En effet, en cherchant à construire la fraternité entre les peuples européens, l'Allemagne semble atteindre ses objectifs. Elle a bien compris qu'il convenait de réussir économiquement mais aussi accompagner socialement cette réussite économique. Mais l'essentiel, selon elle, réside dans la réussite économique qui seule peut permettre l'épanouissement des peuples européens et renforcer leur sentiment d'appartenance à l'Europe.

Aussi, l'ensemble des acteurs de la politique européenne ont-ils prêté une attention particulière à l'élection législative allemande du 22 septembre 2013, conscients de ce que l'avenir de l'Europe s'y jouait en partie. Une Europe qui, selon l'eurodéputé belge du PSE, Marc Tarabella, « continue de diviser les gens, de favoriser la concurrence des uns par rapport aux autres de manière déloyale et d'instaurer le doute et la confusion dans l'esprit des citoyens par rapport au bien fondé de l'intégration ». Certes, Angela Merkel a gagné mais elle va devoir composer autour d'une large coalition avec le SPD.

\section{Encore Merkel! «Une physicienne ne peut pas être foncièrement mauvaise » ${ }^{(14)}$}

Depuis 2005, Angela Merkel a joué un rôle déterminant dans la transformation de l'Union européenne pour faire face à ces crises spécifiques, tout en restant ferme sur un certain nombre de mesures, exigeant que les pays du Sud gèrent leurs économies respectives avec plus de rigueur, avant qu'il n'y ait davantage de solidarité au niveau européen ${ }^{(15)}$.

Ces élections ont un enjeu crucial, dans la mesure où la politique menée par Angela Merkel, que l'on qualifie de politique orthodoxe destinée à lutter contre l'inflation avec l'instauration de l'austérité budgétaire au sein de l'Union européenne, est mal perçue par certains Allemands. Elle aurait pu ainsi mettre Angela Merkel en difficulté pendant ces élections mais ce ne fut finalement pas le cas.

Angela Merkel affirmait elle-même en 1990, peu après la chute du Mur: «J'aurais aussi bien pu adhérer au parti social-démocrate... Je suis simplement allée là où l'on avait le

13 É. Husson, Une autre Allemagne (note 7), p. 137-139.

14 M. Matussek, Nous les Allemands (note 8), p. 78.

15 Cédric Durand (dir.), En finir avec l'Europe, Paris, Éditions La Fabrique, 2013, p. 133-147. 
plus besoin d'aide. » Au vu de son succès dans la CDU, on ne peut que l'approuver. Elle a réussi à apaiser les tensions entre les Allemands de l'Est ${ }^{(16)}$ dont elle est issue et ceux de l'Ouest, de qui elle tient les rênes du pouvoir. Elle est aimée et appréciée de tous. Au sein de la population allemande, dans son parti, elle est plébiscitée, comme le souligne l'historien Hagen Schulze: « Nous avons trop peu tiré de l'unité allemande. Nous sommes en train de tirer quelque chose de plus. L'élection de cette "Chancelière de l'Unité" qui se concevait elle aussi comme telle, a été un premier pas dans cette direction " ${ }^{(17)}$.

Pourtant, un an après sa première élection, au deuxième semestre de l'année 2006, la situation de l'Allemagne était loin d'être assurée. Tout comme ses prédécesseurs, Angela Merkel a connu des moments très difficiles ${ }^{(18)}$. La popularité de la Chancelière et celle de la grande coalition sont alors descendues au plus bas dans divers sondages ${ }^{(19)}$, pour avoir pris trop de mesures contraignantes. Cependant, s'érigeant en pôle de stabilité pour son parti et en personnalité confiante pour l'Union, elle s'en est bien sortie.

C'est cette image d'une femme dynamique et déterminée qui a prévalu jusqu'à nos jours. L'essentiel de la campagne pour les élections de 2013 a été axé sur la personnalité d'Angela Merkel et ses succès politiques, économiques et sociaux ${ }^{(20)}$. Le taux de chômage est descendu à un seuil acceptable grâce à l'intelligence de la Chancelière, encourageant l'entreprenariat individuel, la réforme de la retraite, ainsi que l'allongement de la durée de cotisation de 65 à 67 ans. Au delà de ces avancées, il faut également reconnaître qu'Angela Merkel bénéficie aussi d'un encadrement social bien structuré, ainsi que d'une opinion publique qui apprécie l'ordre et la sobriété. Sa facilité à comprendre les grands défis du $\mathrm{XxI}^{\mathrm{e}}$ siècle et sa lecture lucide de diverses situations vont précipiter son extraordinaire ascension politique. Véritable symbole de l'unification, Angela Merkel l'aurait reconnu dans une réponse adressée un soir à un journaliste: «...Non, je suis un résultat de l’Unité de l'Allemagne».

C'est elle qui dirige et le pays l'approuve. Tout le monde est plus optimiste depuis que la Chancelière gouverne. Selon un extrait de journal électronique inséré dans Nous les Allemands de Matussek, «Une physicienne ne peut pas être foncièrement mauvaise » ${ }^{(21)}$. Et Tymothy Garton, un journaliste du Guardian, d'attester que « L'Allemagne d'aujourd'hui est due à cette nuit électorale indécise de 2005 où Merkel a été portée au pouvoir».

16 Jacques-Pierre Gougeon, Allemagne: une puissance en mutation, Paris, Gallimard, 2006, p. 208-211.

17 Cité par M. Matussek, Nous les Allemands (note 8), p. 87.

18 J. КuвоsсH, «La place de l'Allemagne dans l'Union européenne » (note 7), p. 30; Bertrand GALLICHer, L'Allemagne au pied du mur, Paris, Éditions de la Table ronde, 2009, p. 250.

19 Jean-Paul Pica Per, Angela Merkel, la femme la plus puissante au monde, Paris, JC Gawsewitch Éditeur, 2010, p. 174-175. En mars 2006, les acteurs de l'économie et de l'administration estimaient à hauteur de $77 \%$ que Merkel était un chef de Gouvernement « fort ». Très vite la tendance s'est infléchie jusqu'en août 2006 déjà, le nombre de ces derniers avait progressé de $31 \%$ tandis que celui des premiers avait diminué d'autant. Alors que $40 \%$ seulement des industriels donnaient en mars 2006 une mauvaise note à la politique économique de la grande coalition, ils furent à partir de l'été de cette année $70 \%$ à le faire.

20 J.-P. PicAper, ibid., p. 177-179.

21 Expression tirée d'un journal électronique insérée in Nous les Allemands de M. Matussek (note 8), p. 78. 
De tradition libérale, Angela Merkel a mené une campagne stratégique de gauche pour attirer l'électorat social-démocrate. Elle a su adapter son discours à ce que souhaite le peuple et par rapport à ce qu'une majorité électorale pourrait désirer. En réalité, Angela Merkel a été extrêmement habile en devançant l'opposition sur un certain nombre de sujets sociaux tels que des garanties minimales portant sur le niveau des salaires, le pouvoir d'achat, la qualité des services publics et la qualité de l'éducation. Sur l'un des sujets environnementaux d'arrêt du nucléaire après le drame de Fukuyama, Angela Merkel a privé les Verts de l'essentiel de leur programme électoral tout en " happant » leur électorat. Au niveau de l'épineuse politique européenne de l'Allemagne et de sa politique internationale, l'acceptation de la Chancelière de se joindre à l'appel d'une douzaine de pays du G20 pour « une réponse internationale forte à la crise syrienne ", confirme le statut de nouvelle grande puissance mondiale que devient l'Allemagne. La campagne des législatives en Allemagne ayant été attentivement suivie par les eurodéputés, les résultats conduisent à une nouvelle perspective sur sa position européenne.

\section{Analyse du vote: l'Allemagne à la redéfinition d'une nouvelle orientation politique}

Au début de la campagne, les sondages ont parfois annoncé la victoire du SPD en coalition avec les Verts. Les parlementaires européens ont développé quelques analyses à ce propos. Pour les socialistes du PSE, l'hypothèse de la victoire du SPD lors de cette élection n'aurait rien changé à la politique allemande si ce n'est que l'orientation socialiste aurait été renforcée à travers les différents accords politiques en cours dans l'Union. Par conséquent la démocratie aurait été renforcée, en même temps qu'aurait décru le niveau d'euroscepticisme allemand.

Selon certains parlementaires européens du PPE, la réélection d'Angela Merkel ne changera également pas grand-chose. Sur les choix européens importants, en Allemagne comme dans les autres grands États membres de l'Union, il existe un consensus entre les partis de gauche et ceux de droite. Ainsi, les représentants de la CDU/CSU, du FDP, du SPD et des Verts affirment-ils que l'orientation politique européenne actuelle prise par l'Allemagne ne changerait pas pour autant. En cas de victoire du SPD, l'orientation politique aurait eu une tendance un peu plus sociale et écologique. Mais selon ses représentants, les grands choix européens resteront identiques dans la mesure où le score réalisé par le SPD (25,74\%) ne lui donne aucune clef d'influence. En revanche, la tendance serait allée vers plus de fermeté en cas d'alliance entre le parti d'Angela Merkel et le FPD, avec un risque tout de même si la coalition s'était élargie aux partis extrémistes.

Il paraît évident à l'eurodéputé du PPE Alain Lamassoure que, si la coalition sortante (CDU/FPD) avait été reconduite, l'Allemagne aurait continué à suivre la même orientation politique. Avec tout de même un élément positif car un certain nombre de sujets qui sont actuellement gelés au niveau européen pour ne pas gêner les élections allemandes, vont trouver un aboutissement. C'est le cas notamment du grand sujet portant sur les accords budgétaires ${ }^{(22)}$ qui sont en train d'être conclus et qui nécessi-

22 http://www.lemonde.fr/economie/article/2013/11/12/accord-sur-le-budget-europeen_3512079_3234. html. 
tent d'être complétés par une réforme du système de ressources du budget européen. Le SPD et les autres partis étant favorables, il y aura donc des avancées dans ce sens.

Aux yeux des parlementaires de sensibilité socialiste PSE, le score de 4,9\% réalisé par les libéraux est salutaire car il va rouvrir la voie à une alliance souhaitée entre la CDU/ CSU qui a brillamment gagné ces élections et le SPD dont l'essentiel du programme s'articule autour du thème de l'harmonisation sociale. Même si la mise en œuvre de cette réforme peut prendre du temps, la question du salaire minimum est également une exigence que la Chancelière doit porter jusqu'au niveau européen. L'autre vérité du miracle allemand constitue le revers de la médaille dont on peut difficilement se réjouir: le règne des petits jobs et de la précarité. En effet, l'Allemagne emploie dans des conditions critiquables de jeunes travailleurs provenant d'autres États européens, notamment des ouvriers roumains et bulgares payés 3 à 4 euros de l'heure.

Ainsi, les eurodéputés socialistes espèrent-ils qu'à l'issue de ces élections allemandes soit envisagé un revenu minimum, comme moyen d'intégration des pays du Sud. Car c'est au moment où on accompagne le peuple que la démocratie est légitimée et qu'elle peut se prémunir contre les mouvements euphoriques qui favorisent l'ascension de partis extrémistes.

Pendant la campagne, l'une des questions qui se posait régulièrement était celle de savoir quelle coalition Angela Merkel constituerait si elle était réélue pour un troisième mandat? Aurait-elle une majorité absolue? Une majorité avec les libéraux? Ou une grande coalition? Suite aux résultats, c'est donc l'option de la grande coalition qui va prévaloir comme ce fut le cas entre 2005-2009. Est-ce à dire pour autant que l'orientation politique définie par la Chancelière sera remise en cause?

La réflexion à cette question et l'analyse du score réalisé par le SPD conduisent à penser qu'il n'y aura pas une véritable alternance. Pour Jean-Louis Cottigny eurodéputé du groupe PS-PSE, il y aura certes une nouvelle inflexion vers plus de tolérance envers les partenaires européens en crise, sans pour autant modifier de fond en comble le rapport de l'Allemagne à l'Union européenne, le SPD n'étant pas véritablement en position d'influencer la politique d'Angela Merkel. Par conséquent, la Chancelière affranchie de toute pression laissera le temps aux États en crise pour atteindre leurs objectifs de redressement économique. De surcroît, le SPD garde un mauvais souvenir de la coalition 2005-2009 dont il est sorti exsangue. À Angela Merkel de se montrer collaborative et humble dans la nouvelle coalition afin de ne plus heurter le SPD, qui l'avait considérée comme trop rigide durant la dernière coalition.

Tout compte fait, le SPD est loin d'apparaître impuissant face à Merkel dans cette nouvelle coalition, même s'il n'a rien à y gagner. Il a été en effet privé d'importants portefeuilles ministériels qu'il désirait, à l'exemple du ministère des Finances qui ne lui a pas été proposé. Le seul avantage qu'y trouve le SPD, c'est de préparer le terrain et de travailler sur son positionnement stratégique dans les Länder allemands, en vue d'élections futures. Implanté dans le pays en tant que deuxième force politique importante, le SPD a déjà gouverné avec la CDU/CSU et a l'habitude des grandes coalitions. Compte tenu des idées qu'il défend, il sera impossible d'ignorer ce parti qui certainement pèsera sur les futures élections grâce à la personne de Martin Schulz, l'actuel Président du Parlement européen dont on connaît l'attachement à la construction européenne et qui peut devenir un candidat potentiel à la chancellerie. 
Cependant, après ces élections, quel va être le positionnement du nouveau gouvernement? Tout va dépendre des alliances qu'Angela Merkel cherchera à établir. Va-t-elle vouloir obtenir plus d'influence, c'est-à-dire aller plus loin dans la construction européenne mais à travers une conception européenne gouvernementale comme ce fut le cas pour le paquet fiscal, ainsi que pour les accords dans la zone euro destinés à renforcer l'Europe institutionnelle, comme seul moyen pour l'Allemagne de faire jouer son importance politique dans l'Union? Politiser la zone par le biais d'accords intergouvernementaux comme le cas du paquet fiscal, tout en tenant compte des avis du SPD? Car même si Angela Merkel est renforcée dans sa position, faire une politique qui tienne compte de plus d'harmonisation sociale et institue le salaire minimum tel qu'il est stipulé dans le programme du SPD, devait être l'une des clefs de réussite de la nouvelle coalition en voie de formation.

\section{Une eurythmie entre intellectuels choisis: Alternative für Deutschland}

L'insatisfaction à l'égard de la politique européenne menée par Angela Merkel, ainsi que la montée de l'europhobie et l'euroscepticisme ont incité certains intellectuels à imaginer des solutions alternatives en créant le nouveau parti Alternative für Deutschland (AfD). Composé de professeurs, d'ingénieurs et de journalistes experts en économie, son objectif est celui de « s'installer durablement dans le paysage politique de la zone euro et de veiller à la dissolution contrôlée de cette zone " ${ }^{(23)}$. Classé du côté de la droite conservatrice, son appartenance à la tendance populiste est discutée. Ce parti extrémiste, qui prétend n'être ni de gauche ni de droite, se caractérise par une tendance au repli national.

En France, en 1999, six députés européens ont été élus dans le parti des chasseurs ${ }^{(24)}$, lequel n'existe plus aujourd'hui. C'est la preuve du rôle minimaliste auquel de tels partis peuvent être assignés dans le paysage politique. Aussi, la configuration de ce parti « intellectuel » contre la zone euro est un prototype à classer dans l'ordre de ce que l'on peut qualifier de folklore électoral. S'il a pu réaliser un score final de 4,7 \%, c'est parce qu'il est parvenu à mobiliser les électeurs volatiles, sans aucun attachement à un parti quelconque, si bien qu'il a pu égrener quelques voix aux libéraux (FPD) et à la CDU. Cependant, ce qui est important et positif, c'est qu'il n'existe pas en Allemagne de grands partis populistes démocrates comme c'est le cas dans la plupart des États de l'Union européenne à l'exception de l'Espagne et du Portugal...

Ainsi, le fait que l'AfD ne soit pas représenté au Bundestag n'en est pas pour autant surprenant. En effet, la République fédérale a mis en place une loi électorale très contraignante pour les petits partis qui fait obstacle au morcellement politique. C'est ce qui avait été fatal à la République de Weimar, laquelle, en favorisant la multiplicité des partis et des groupuscules politiques, avait contribué à l'accession du fascisme au pouvoir. Seuls les partis ayant obtenu au moins trois sièges par le scrutin uninominal

23 http://www.latribune.fr/actualites/economie/union-europeenne/20130923trib000786589/allemagnele-parti-anti-euro-a-ratisse-large.html.

24 André Goustat, La parole aux terroirs. Le manifeste des chasseurs, Paris, Éditions du Rocher, 1994, 140 p.; «Le Lobby des chasseurs contre l'Europe », Le Monde, 26 février 1992; Christophe TraïNI, Les braconniers de la République, Paris, Presses Universitaires de France, 2003, 209 p. 
ou $5 \%$ des voix au niveau national ont vocation à entrer au Bundestag ${ }^{(25)}$. Cette disposition a pour finalité d'assurer la constitution de majorités stables, avec des partis bénéficiant d'une réelle représentativité.

Même si l'AfD a réussi à prendre au parti d'Angela Merkel, aux Verts et aux libéraux les voix des citoyens mal informés, il ne jouera pas, selon Alain Lamassoure, un rôle fondamental dans la construction des positions européennes au sein du gouvernement allemand $^{(26)}$. De toute manière, il y a un large consensus entre le SPD, la CDU/CSU, les Verts et le FPD selon lequel l'Allemagne est très bien ancrée dans l'Union européenne, que c'est une bonne chose et que l'euro est la devise qui peut engendrer l'économie de l'avenir. On ne voit donc pas quelle aurait été l'influence d'un petit parti comme l'AfD qui ne peut permettre aucune participation collective. De surcroît, il aurait été difficile à Angela Merkel d'envisager une coalition avec ce parti sans risquer d'affaiblir sa propre orientation politique et les valeurs qu'elle défend.

Un parlementaire belge du PSE, Marc Tarabella, réfléchissant aux partis antieuropéens tels que l'AfD souligne: "L'Allemagne n'est pas un problème mais une solution à tous les problèmes, parce qu'elle est plus sensible face à certaines questions (la solidarité européenne) qu'à d'autres (la politique économique rigoriste). Aujourd'hui, ce qui manque en Allemagne ou au sein de l'Union, c'est le retour aux valeurs de solidarité et de développement commun car les Européens ont besoin d'être rassurés par une Europe qui protège, qui rassure et ce n'est pas encore le cas. C'est la raison pour laquelle il faut changer de cap pour décider de politiques mieux concertées entre les politiques et les citoyens " ${ }^{(27)}$.

La seule crainte que l'on aurait pu avoir si ce parti entrait au Bundestag, selon ce même eurodéputé, aurait été qu'il génère le basculement ou la radicalisation de partis majoritaires démocrates. Cependant, la mémoire de l'expérience du passé va toujours faire obstacle aux partis extrémistes non consensuels. Ces partis peuvent exister pour soutenir des majorités au Parlement mais ils n'auront aucun grand rôle à y jouer. Loin s'en faut! Toutefois, l'influence de l'Allemagne au sein de l'Union européenne, son dévouement et son investissement corps, âme et esprit, interpelle et attire l'attention de tous.

\section{Impact de l'élection législative allemande sur l'Union européenne}

Même s'il n'y avait pas d'incertitude sur le résultat, cette élection allemande a eu un fort retentissement, du moins sur les positions prises par les eurodéputés au sein de l'Union européenne. Notamment sur la question du redressement économique qui a conduit aux mesures d'austérité en vigueur. Mais ce n'est pas tout. Sa position particulière au sein de l'Union passée au crible, l'Allemagne exportatrice nette et bénéficiaire du grand marché européen, par rapport à d'autres États membres, crée du déséquilibre macroéconomique. Certains voient dans l'excédent commercial allemand la cause du déficit de leur propre pays, au mépris de l'intérêt commun européen qui exigerait un retour à une situation normale.

25 Jean-Claude CA PÈLe, L’Allemagne hier et aujourd'hui, Paris, Hachette, 2012 (8éd.), p. 84-87 et 88-89.

26 Témoignage d'Alain Lamassoure.

27 Témoignage de Marc Tarabella. 
Pendant la campagne, l'une des préoccupations des eurodéputés a été celle de mesurer la portée de cette élection sur l'Union européenne. Jean-Paul Gauzès, eurodéputé français du PPE, pense que cette élection n'aura pas beaucoup d'impact sur l'Union. Néanmoins, s'il y avait une forte poussée du SPD et des Verts, et que la CDU en sorte affaiblie, ce résultat aurait pour conséquence de réorienter à gauche la politique de l’Union européenne. Au niveau du Parlement, 60 \% des députés européens appartiennent à des gouvernements qui sont au pouvoir dans leur pays, qu'ils soient de droite ou de gauche. Ainsi défendent-ils généralement la ligne politique définie par leur gouvernement national, plutôt que d'essayer de trouver un intérêt européen commun.

Les résultats des élections européennes peuvent par contre modifier l'équilibre politique au Parlement de Strasbourg de manière beaucoup plus considérable, dans la mesure où l'Allemagne détient le plus grand nombre d'eurodéputés. Il reste à savoir si ce sera la CDU/CSU ou le SPD qui sera dominant. Le parti majoritaire déterminera alors l'orientation politique européenne, soit vers une politique plus sociale et modérée, soit vers une politique libérale et radicale où règne le «tout économie » ${ }^{(28)}$.

Selon Évelyne Gebhardt, eurodéputée allemande du PSE, si Peer Steinbrück adversaire social-démocrate d'Angela Merkel lors des élections législatives allemandes l'avait emporté, il aurait fait en sorte que la politique fiscale soit plus cohérente et plus dure vis-à-vis des banques. La politique d'Angela Merkel manque de concrétisme dans la mesure où la Chancelière ne cesse de tâtonner en voulant réguler le secteur des finances, alors qu'il faudrait que se dégagent des propositions plus tangibles ${ }^{(29)}$.

Les mesures d'austérité imposées par la Chancelière et Bruxelles se répercutent par ailleurs dans les États membres de l'Union. Angela Merkel, reconduite à l'issue des élections législatives allemandes, n'entendra pas changer de cap et va poursuivre sa politique économique d'austérité dans la zone euro, puisque celle-ci n'a eu aucune conséquence sur son propre sort politique. La preuve en est qu'elle a été réélue et reste toujours plus que jamais déterminée à poursuivre cette même orientation politique. Dans le cas contraire, estime Évelyne Gebhardt, Angela Merkel aurait été sanctionnée et écartée. Néanmoins, les effets de cette politique au niveau de l'Union européenne restent à déterminer.

En réalité, cette politique ne devrait pas s'appliquer d'emblée à tous les États membres de l'Union. Elle est avant tout destinée à redresser l'économie de certains pays qui pendant longtemps ont vécu au-dessus de leurs moyens. L'Allemagne, la France, le Pays-Bas, le Danemark, par exemple, auraient peut-être pu en être dispensés parce qu'ils ne sont pas concernés.

D'après les analyses faites par Alain Lamassoure, la situation est plus critiquable en Espagne, qui a connu une crise immobilière sans précédente. Pendant longtemps, les Espagnols ont mis en chantier plus de logements qu'en France, en Allemagne et en Italie réunies. Soudain, il est apparu qu'il n'y avait plus de possibilités de vente des logements nouvellement construits, faute de demandes. Tout s'est effondré et l'Espagne a été mise en faillite. Les partenaires de l'Espagne ont souhaité lui venir en aide à condition qu'elle soit capable de rembourser les dettes qu'elle avait contractées. 
La situation est différente chez les Grecs, qui considèrent que l'obligation qui leur a été faite de se "serrer la ceinture " et de faire des efforts économiques a été causée par l'Allemagne. Alors que ces mesures d'austérité auraient été deux fois pires sans l'Allemagne. Selon Alain Lamassoure, dans la fonction publique grecque, au moins un tiers des fonctionnaires rémunérés ne se rendent pas à leur travail. Dans la constitution grecque, il y a une disposition selon laquelle les armateurs sont dispensés de toute sorte d'impôt. Alors que ces armateurs sont des milliardaires, contrôlant presque $15 \%$ du budget de la Banque mondiale ${ }^{(30)}$.

Pourtant, l'Allemagne était encore disposée à porter son aide à la Grèce, à condition que, même si la moitié de la dette n'est pas soldée, l'État grec fasse l'effort de cesser de payer les fonctionnaires non productifs afin de payer les seuls actifs.

Les Allemands, les Néerlandais aussi bien que les Français ont stigmatisé le système grec, car ceux qui étaient contraints de faire des sacrifices, rendus nécessaires par leurs propres erreurs, en rendaient l'Allemagne et l'Union européenne responsables.

Les conséquences de cette situation se mesureront lors des prochaines élections européennes, si, par exemple, les cinq grands États de l'Union décident de se soustraire à la solidarité. Les pays en crise seraient obligés de sortir de l'Union pour faire face à leurs difficultés. Mais en aucun cas, cette mesure d'austérité n'a pu influencer la politique intérieure de l'Allemagne.

La majeure partie des eurodéputés considèrent que l'Union est en train de sortir de la phase la plus difficile de la crise dans la mesure où les Allemands se rendent compte qu'il est de leur intérêt d'atteindre un taux de croissance plus élevé dans leur propre pays. Or jusqu'à présent, la stratégie de croissance allemande reposait essentiellement sur l'exploitation de ses performances industrielles et commerciales. Le marché international qui s'était montré florissant pendant de longues années montre ses limites aujourd'hui car les principaux clients de l'Allemagne, comme la Chine, l'Inde et le Brésil, lui achètent désormais beaucoup moins. De surcroît, l'économie européenne étant en récession, la croissance enregistrée est nulle. Les Allemands en ont ainsi profité pour rééquilibrer leur croissance et recommencer à distribuer le pouvoir d'achat à leurs concitoyens. Les entreprises ont ainsi pu augmenter les salaires, en particulier dans le secteur automobile ${ }^{(31)}$.

Selon les promesses de campagne d'Angela Merkel, le gouvernement fédéral va se lancer dans une campagne d'investissement public. De ce fait, l'Allemagne et les autres pays qui ont des marges de manœuvre importantes pourront les utiliser pour soutenir leur croissance économique et tirer vers le haut les pays de l'Europe du Sud qui ne cessent de creuser leur déficit, lequel les contraint à subir la cure d'austérité.

Si l'on s'en tient aux réflexions des élus du PPE tels qu'Alain Lamassoure, les premiers résultats sont déjà visibles depuis un an dans le cas de l'Espagne et depuis quelques mois dans le cas de la Grèce et du Portugal. Ces pays ont rétabli leur commerce extérieur

30 Le Monde du 13 juin 2013: " Le mardi 11 juin dernier à 23h15, le gouvernement dirigé par le Premier ministre Antonis Samaras a brutalement coupé les antennes de la Radio et Télévision ERT. Selon ce dernier, la télévision publique est étrillée comme un symbole de corruption et de clientélisme. Il s'agit en effet d'un entrelacs de conflits d'intérêts entre bailleurs de fond, propriétaires de chaînes de médias et le pouvoir qui tant dans les médias publics que dans les médias privés arrive à imposer leur vue. » 
par rapport à l'Allemagne et redeviennent progressivement compétitifs. Grâce aux efforts fournis, certains produits espagnols deviennent presque plus compétitifs que les produits allemands. On assiste ainsi à une sortie de crise par le haut. Cependant, les avis donnés par les eurodéputés du PSE attestent du contraire, dénonçant les effets négatifs du plan de rigueur.

\section{Conclusion}

S’il était question d'un choix idéologique dans cette élection, ce n'étaient pas les programmes défendus par l'un ou l'autre des candidats qui ont prévalu. Et même si les attitudes et les réseaux de souvenirs auxquels étaient attachés les candidats y ont été pour quelque chose, la personnalité d'Angela Merkel et son succès politique ont représenté une valeur ajoutée. Les Allemands sont devenus normaux et pragmatiques. L'Allemagne, comme l'écrivait avec lyrisme dans son dernier livre Steve Crawshaw, du journal The Indépendant, est devenue depuis un certain temps « une Patrie plus simple " ${ }^{(32)}$. C'est-à-dire qu'un bouleversement s'est produit en profondeur depuis longtemps et que ses effets arrivent désormais à la surface. En effet, les Allemands ont cherché à se réhabiliter par le «tout économie ». Il s'agit donc d'une "opération de rédemption ». Issues de l'air du temps, les idées reçues sur l'Allemagne sont loin d'être immémoriales.

Cet article a ainsi essayé de fournir un éclairage distancié et approfondi mais non exhaustif sur ce que l'on sait, ou croit savoir sur l'Allemagne. Les préjugés et les ressentiments s'atténuent aujourd'hui à son égard. Grâce au succès économique de celle-ci, à sa place dans la construction européenne et à sa volonté de rester un État de droit ${ }^{(33)}$. L'intégration des immigrés est également acquise, faisant de l'Allemagne « un pays de l'intégration », et l'Europe n'est plus pour les Allemands une affaire de cour. Elle est devenue une question de raison.

L'Allemagne n'oscille plus entre la célébration de ses acquis politiques, sociaux et la quête de repères, susceptibles d'ancrer son identité politique et géostratégique née de l'unification. Désormais, elle est à la recherche d'une nouvelle identité de grande puissance. Mais en attendant, elle s'affirme, elle provoque et innove ${ }^{(34)}$.

\section{Résumé}

Les élections législatives allemandes du mois de septembre 2013 ont représenté un enjeu crucial. La politique menée par Angela Merkel, destinée à l'instauration de l'austérité budgétaire au sein de l'Union européenne, était mal perçue par certains Allemands. Elle aurait pu mettre la Chancelière en difficulté. Mais ce ne fut finalement pas le cas. La vision des eurodéputés nourrie par les débats, aussi bien que les enjeux

32 Steve Crawshaw, Easier Fatherland: Germany and the Twenty-First Century, Londres, Continuum, 2004.

33 Bettina Mrosowski, Savoir vivre avec les Allemands - Petit guide interculturel, Paris, L'Harmattan, 2003, p. 101-109

34 Claire Demesmay, Daniéla Heimerl, Allemagne, une mystérieuse voisine: portrait en vingt tableaux, Paris, Éditions Lignes de repères, 2009, p. 10-13. 
qu'ils ont fait naître, à savoir les problèmes qui demeurent en suspens et qui posent le jalon des analyses postélectorales, d'une part, et l'implication de l'Allemagne dans le processus de construction de l'Union à partir de réflexion sur son histoire, d'autre part, sont autant d'éléments qui donnent la mesurabilité de l'impact de ce scrutin pour l'avenir de l'Union européenne. Peut-on affirmer que la responsabilité de l'Allemagne, en laquelle l'Europe perçoit une partie d'elle-même, favorise le succès collectif de l'ensemble des États membres? En réalité, l'Allemagne cherchant à construire la fraternité entre les peuples européens semble atteindre ses objectifs. Elle a compris qu'il convenait de réussir économiquement mais aussi d'accompagner socialement. Car seule la réussite économique peut permettre l'aboutissement de l'Europe politique.

\begin{abstract}
The German Parliamentary elections in September 2013 represented a crucial stake. The policy led by Angela Merkel, intended for "The implementation of very strong budget austerity" within the European Union, was often misunderstood and badly viewed by some Germans. It could have brought the German Chancellor in trouble. But finally it was not the case. The goal and vision of the Members of the European Parliament, nurrished by the debates as well as the issues and the expectations they raised, namely the problems which remain unresolved and which are the milestone for the post-electoral analysis on the one hand, and Germany's involvement in the process of the building of the Union starting with reflection on its history on the other hand, are the many factors measuring the impact of this ballot for the European Union's future. Can we assume that the responsibility of Germany, to which Europe may partly identifies itself, promotes the collective success of all Member States? In fact, Germany, searching the way to create brotherly bonds among the European people, seems to achieve its goal: it was considered appropriate to make success economically but also accompany socially. For only the economic success may lead to a successful realisation of a political Europe.
\end{abstract}

